\title{
Methods for sustainable management of secondary resources
}

\author{
A. Pehlken, M. Rolbiecki, A. Decker \& K.-D. Thoben \\ Institute of Integrated Product Development, Bremen University, \\ Germany
}

\begin{abstract}
Dumping secondary raw materials is already forbidden by legislation in Europe. Due to limited resources, especially in high-tech products or the dependency on other countries who provide specific minerals or metals, efficient recycling processes are improving to guarantee a sustainable management of secondary resources with replacing primary resources.

We can save our raw materials by either reducing the consumption of primary raw materials or the increased use of secondary raw materials. Processing waste materials ensures the availability of secondary resources. To evaluate the potential of the reusability of materials the knowledge of material flows of recycling processes and their characteristics is essential.

For example, the knowledge of the quantity and quality of material flows gives an indication for the assessment of the environmental process performance. The combination of Material Flow Assessment (MFA) and Life Cycle Assessment (LCA) may help to improve sustainable resource management and makes environmental decision making easier.

This paper will give an overview on the required information received from recycling processes to build up an environmental management system to serve as a tool for sustainable material management. The main focus lies on the material characteristics, uncertainties and the material's future potential to replace primary resources.
\end{abstract}

Keywords: material flow assessment, life cycle assessment, sustainable resource management, uncertainty. 


\section{Situation of primary and secondary resources}

Primary raw materials are being exploited and especially Europe depends on imports from other continents. China for example produces $95 \%$ of all rare earth concentrates which are commonly used in "high tech" products. Motors for hybrid cars cannot be manufactured without rare earth dysprosium, drilling tools with tungsten tips are vital for the production of compact mobile phones and rare earth is commonly used in high performance permanent magnets. Furthermore, China is the top one mining region for other metallic minerals like Antimony (87\% of the world production), Tungsten (84\%), Gallium (83\%) and Germanium $(79 \%)$ for example. To guarantee stable production of materials with these ingredients some countries develop "stockpiling-policies" and have always specific raw materials in stock. This is extremely important if the political situation remains unstable in some countries, like Congo for example as the number one world producer of cobalt (36\%). Additional there are "critical raw materials" defined which are dependent on the supply and the geological resources and reserves of each material. For example a material is called "critical" if over $60 \%$ of its resources gain from one country. In this case the stable supply of this material cannot be guaranteed because it relies on the political situation of one country. Another example of a critical material is the fact that some geological resources last only for a few decades. Therefore, the effort to find solutions to either recycle or substitute this material is very high. According to Ziemann et al. the metals Antimony, Chrome, Germanium, Gold, Niobium can be identified as materials with a high potential to find solutions for substitution (Ziemann et al. [1], Commission of the European Communities [2]).

The resource-efficient and recycling-based industry will strike a balance between dematerialization and rematerialization to support the availability of secondary resources (EPA [3]). These secondary resources will enter the common production processes as substitute for primary raw materials. This implies that countries with well managed recycling process will be less dependent on imports (Braungart and McDonough [4]).

\section{Material flow assessment and LCA in waste management}

Life Cycle Assessment (LCA), which is used to assess the environmental impacts of products from cradle to grave, is increasingly being applied to the evaluation of waste management strategies. It should be noted however that there is a fundamental difference between the life cycle boundaries of products and wastes. The life cycle of a product starts with the extraction of raw materials (through activities such as mining, logging, etc.) and ends with the final disposal of a product. The life cycle of a waste on the other hand, starts when a material is discarded into the waste stream and ends when the waste material has either been converted into a resource (such as recycled material or recovered energy) or, when it has been finally disposed of [5]. 
Since 1992 the Society of Environmental Toxicology and Chemistry (SETAC) is organizing workshops focused exclusively on uncertainties in Life Cycle Assessments (LCA) (Fava et al. [6]). Modelling recycling processes and the assessment of uncertainty are firmly connected; otherwise the model provides a lack of reliability due to parameter uncertainties. An interest on the credibility of process modelling due to the performed decisions based on the LCA is evident (Pennington et al. [7]). Within recycling processes the known and unknown uncertainties become important parameters to consider. Uncertainties can have many sources and Heijungs and Huijbregts [8] state that the outcome of an LCA is affected by various types of uncertainty, such as parameter, scenario, and model uncertainty. The uncertainty by itself has to be addressed at three places: the input side, the processing side and the output side. The variation of parameters is not always known and coincidental. For example there is data for which no value is available, data for which an inappropriate value is available, and data for which more than one value is available. Due to the fact that residues always vary in their composition and material flow only data ranges can be used as input parameter. A simulation of recycling processes is therefore often difficult. Notten and Petrie [9] substantiate the statement that "different sources of uncertainty require different methods for their assessment". Therefore, the model relies not only on the quality of the process data but also on the uncertainty assessment. For modelling purposes the acceptable level of uncertainty has to be defined (Clyde and George [10]).

Modelling solid waste recycling processes is a challenge because there is few specific data available. Various materials are firmly connected to each other that make an ideal separation success nearly impossible. Useful information can be accumulated with data received from particle size distribution. Assessing the information of the separation for modelling purposes with the availability of data ranges and identifying uncertainties is the focus of this paper.

The management of product life cycle includes the best knowledge of processes and their system boundaries. All recycling processes have in common that their input material has already had a whole life cycle and makes it therefore harder to find exact input data for the LCA of the recycling process. Attention has to be paid to uncertainties in performing LCAs in recycling processes. These days life cycles of secondary resources are no longer within the system boundary from "cradle to grave"; instead we have to look at a life cycle from "cradle to cradle".

\section{Modelling recycling processes}

Modelling recycling processes offers the potential of identifying the processing steps with regard to the main material flows and emissions to reduce the environmental impact and improve the economics. Simulation has to be made available for further investigations. The major problems are the uncertainties on the material composition within the material flows, and in the recycled products; especially regarding their characteristics like size, material combination and distribution. Due to high dynamic uncertainties a complete Life-Cycle 
Assessment cannot be performed for many recycling processes, but Life-Cycle Thinking may be applicable. The processes with the main influence must be identified and evaluated within the model.

A metamodel describes the structure of a model and uses mainly the information generated from processing steps. This results in an abstract combination of the elements of the model and its linkages. Therefore, this technique allows developing the model. Tools to build this model are the LCA software "UMBERTO" to define the mathematical connections between processing steps as well as the programming language "Matlab".

Assessing the information of the processing steps for modelling purposes with the availability of data ranges and the focus on uncertainties is the focus of the research. Uncertainty can be understood as the variation of a parameter in a model. The variation is not known and at random. Modelling recycling processes and the assessment of uncertainty are firmly connected; otherwise the model provides a lack of reliability due to parameter uncertainties. The known and unknown uncertainties of the process become important parameters to consider. Due to the fact that waste materials always vary in their composition and material flows only data ranges can be used as input parameter (Pehlken and Mueller [11]. A simulation of recycling processes is therefore often difficult but a model relies on the quality of the process data. For modelling purposes the acceptable level of uncertainty has to be defined through statistics.

A complete separation of waste material components is not possible but quality standards can be met through defining separation grades as categories (high, medium, low). Therefore, the modelling is not related to specific data (numbers) but rather to data ranges (or quality issues). The modelling can help with the decision making process if the uncertainty of the model has an acceptable level. The acceptable level depends on the aim of the modeler and the model user.

\subsection{Material characterization for modelling purposes}

Information of material characterization is essential for estimating the material distribution and the material flow. Processing steps like comminution and the following separation depend on each other and are essential for the separation success.

The particle size reduction and the liberation of materials during crushing will both effect the recycling of end-of-life products.

Table 1 gives an example on the degrees in separation and crushing. Due to the design and construction of many products it is difficult to measure exact grain sizes of the elements in the product (figure 1). But grain sizes often include the separation of material sizes and there is a correlation between particle size and separation and/or liberation degree. Often the components are well combined and made to never separate by itself due to quality assurance. In future time more attention is paid to an environmental product design that includes the construction of products to have the potential for easy separation. 
Table 1: $\quad$ Degree of separation and crushing (Pehlken and Mueller 11]).

\begin{tabular}{|c|c|}
\hline \multirow{2}{*}{ Separation Degree of materials } & Separated \\
\cline { 2 - 2 } & Partly separated \\
\cline { 2 - 2 } & Not separated \\
\hline \multirow{2}{*}{ Crushing Degree } & Fine \\
\cline { 2 - 2 } & Medium \\
\cline { 2 - 2 } & Coarse \\
\hline
\end{tabular}

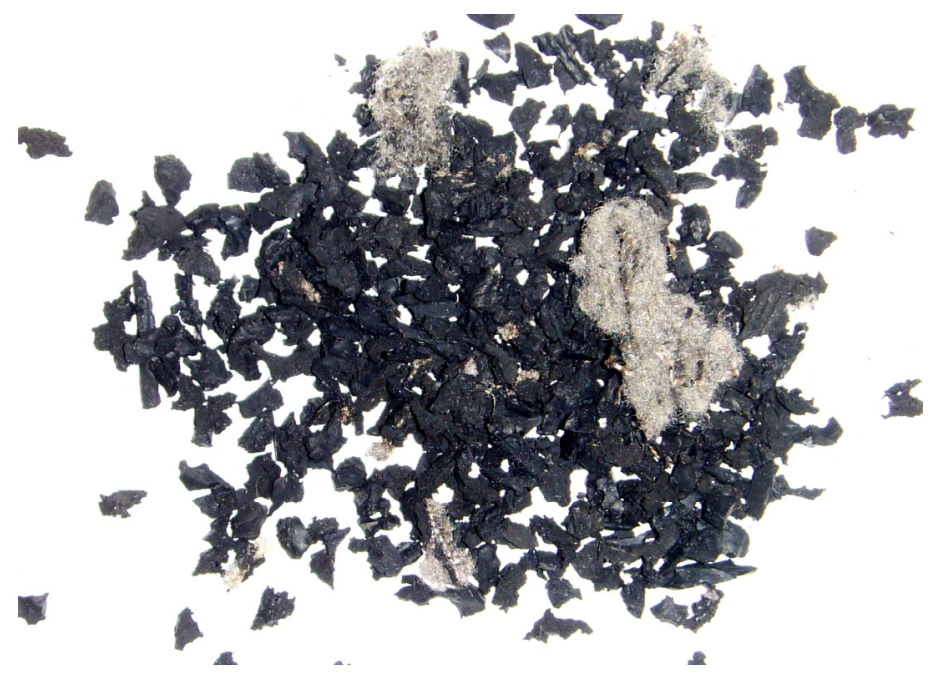

Figure 1: $\quad$ Sample of separated materials from scrap tires after crushing.

\subsection{Uncertainty assessment}

Uncertainties play an evident role in developing a model to assess a recycling process or support a decision based on environmental impacts of processes. Therefore, uncertainties in the process have to be identified and considered as a fact in recycling processes.

Possible uncertainty locations are depicted in Figure 2 and represent only sample locations. Far more locations can be found in processes and have to be taken into account while developing a model. 

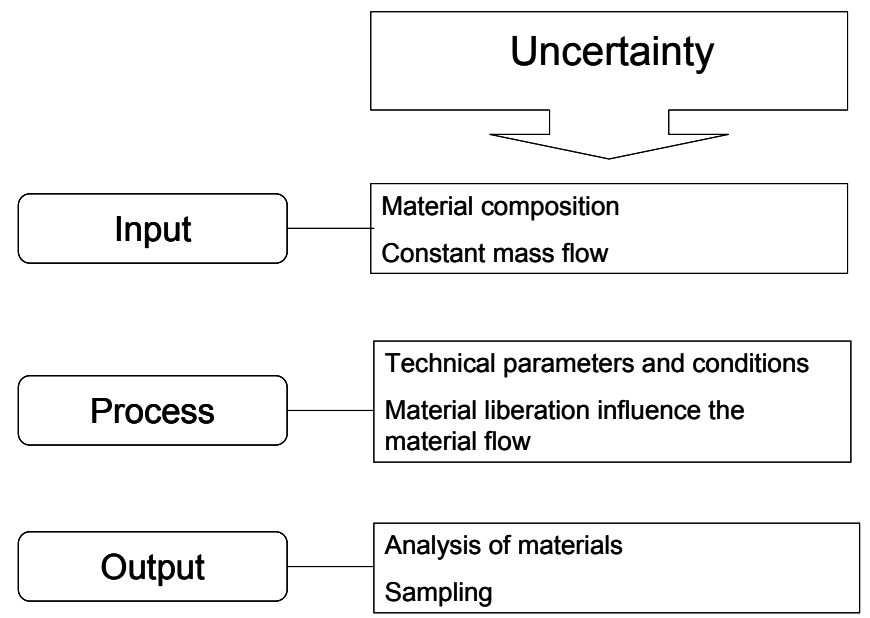

Figure 2: $\quad$ Possible uncertainty locations.

\subsection{Developing a metamodel with respect to material flow characteristics}

Models to predict the material flows AND their properties at the end of the recycling process are essential to close the gap between this information in Life Cycle Management. The developed metamodel (definition: a metamodel represents a model of a model that processes information of a process instead of data) describes a theoretical performance of the model on a high level of abstraction to support the construction of a prediction model for real processes. Complex processes are divided into smaller modules with main effects on the process (modular construction system). Since the functional correlation between modules is often unknown "black-box" models are in use. These "black-box" models have direct or indirect influence on the process step through their input parameters and are therefore important for all following process steps.

To obtain the correlation between input and output parameter of the "black box" laboratory experiments help to identify the relevant process parameter. These results can then be transferred to an operating plant to confirm the developed module transfer function.

As mentioned above the particle size plays an important role in recycling processes. Scrap tires for example are first crushed and then further processed in other process steps that are mainly dependent on the particle size. In this case the "black box" model depicts the modification of particle distribution with their correlation between the single particle size fractions. Various statistical methods have been tested to fit the best transfer function.

Particle size distributions can often follow a normal or Weibull distribution (figure 3). In this case parameter like mean, mean variation or variance of the specific distribution can be easily compared (figure 4) and the data fit can be verified with the Shapiro-Wilk-Test for example. 
Material A

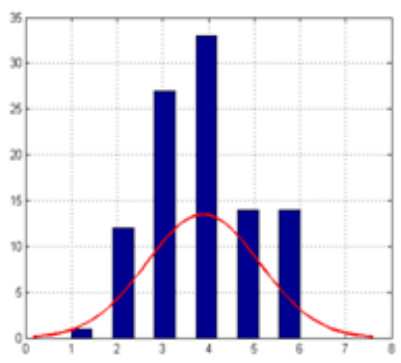

Material B

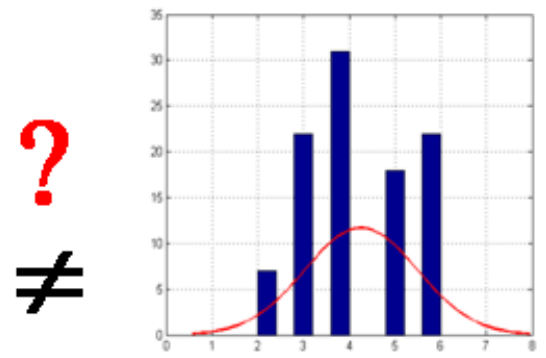

Figure 3: Statistical analysis.

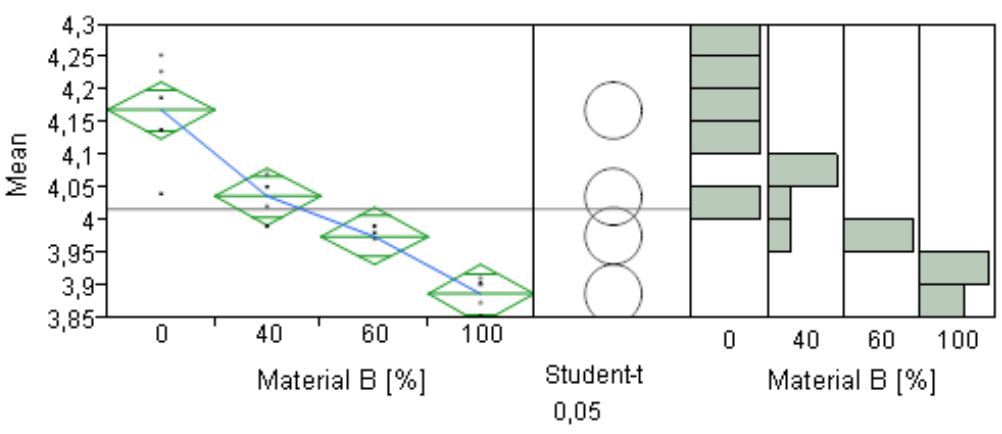

Figure 4: Statistical evaluation of crumb rubber experiments.

Alternatively, it is possible to compare the distances of data points of a distribution to each other in a multi-dimensional space. In n-dimensional space the distances for a distribution of $n$ size parameter can be calculated. The reduction to one parameter (distance) will facilitate the comparison of various distributions.

\section{Conclusion}

The focus on the management of secondary resources will increase the coming years. Due to our limited primary resources and the goal to live in a sustainable environment recycling technologies will improve. Materials Flow Assessment and Life Cycle Assessment can support the determination of the future potential of waste streams entering the recycling process. Some material flows are appropriate to replace primary resources without loss of quality (up-cycling). But other materials are only useful for products with minor quality (down-cycling). Some materials are made to never separate by itself and therefore pure material flows are impossible to achieve. A metamodel that considers the fact of material flows of different material properties may help to evaluate the global recycling 
potential. Therefore, material qualities have to be defined to make an assessment of sustainable management of secondary resources possible.

\section{Acknowledgement}

Part of this work was only possible through funding of the German Research Association (DFG).

\section{References}

[1] Ziemann, S., Schippl, J., Grunwald, A., Schebek, L. (2010) Verfügbarkeit knapper metallischer Rohstoffe und innovative Möglichkeiten zu ihrer Substitution, Rohstoffeffizienz und Rohstoffinnovationen, Fraunhofer Verlag, Stuttgart, Germany

[2] Commission of the European Communities (2008) The raw material initiative - meeting our critical needs for growth and jobs in Europe, Communication from the Commission to the European Parliament and the council, COM (2008) 699, Brussels, Belgium

[3] EPA (9). Sustainable materials management: The road ahead (report and appendix - technical support document, Washington DC: EPA, www.epa.gov/epawaste/inforesources/pubs/vision.htm, accessed February 2010

[4] Braungart, M., McDonough, W., Cradle to Cradle. Remaking the Way We Make Things, New York 2002

[5] EPIC-Environment and Plastics Industry Council and CSR-Corporations Supporting Recycling. Integrated Solid Waste Managements Tools: "Measuring the Environmental Performance of Waste Management Systems". October 2000

[6] Fava, J.A., Jensen, A.A., Lindfors, L., Pomper, S., de Smet, B., Warren, J., Vigon, B., 1994. Life cycle assessment data quality. A conceptual framework. SETAC. Pensacola. USA

[7] Pennington, D.W., Potting, J., Finnveden, G., Lindeijer, E., Jolliete, O., Rydberg, T., Rebitzer, G. (2004). Life cycle assessment Part 2: Current impact assessment practice, Environment International 30, 721- 739

[8] Heijungs, R., Huijbregts, M.A.J., 2004. A review of approaches to treat uncertainty in LCA. iEMS Conference Proceedings: Complexity and Integrated Resources Management. Osnabrueck. Germany

[9] Notten, P., Petrie, J., 2003. An integrated approach to uncertainty assessment in LCA, International Workshop on LCI-Quality 2003. Karlsruhe. Germany

[10] Clyde, M., George, E.I., 2004. Model Uncertainty. Statistical Science (19) No. 1.pp. 81-94

[11] Pehlken, A., Mueller, D.H.; Using information of the separation process of recycling scrap tires for process modelling, Resources, Conservation and Recycling (54) 2009; pp. 140-148; http://dx.doi.org/10.1016/ j.resconrec.2009.07.008 